\title{
Simulation of Proton Beam Effects in Thin Insulating Films
}

\author{
Ljubinko Timotijevic, ${ }^{1}$ Irfan Fetahovic, ${ }^{2}$ Djordje Lazarevic, ${ }^{3}$ and Milos Vujisic ${ }^{1}$ \\ ${ }^{1}$ Faculty of Electrical Engineering, University of Belgrade, 11060 Belgrade, Serbia \\ ${ }^{2}$ State University of Novi Pazar, 36300 Novi Pazar, Serbia \\ ${ }^{3}$ Institute of Nuclear Sciences "Vinca", 11000 Belgrade, Serbia \\ Correspondence should be addressed to Milos Vujisic; vujsa@ikomline.net
}

Received 15 April 2013; Accepted 28 May 2013

Academic Editor: Predrag Osmokrovic

Copyright (C) 2013 Ljubinko Timotijevic et al. This is an open access article distributed under the Creative Commons Attribution License, which permits unrestricted use, distribution, and reproduction in any medium, provided the original work is properly cited.

Effects of exposing several insulators, commonly used for various purposes in integrated circuits, to beams of protons have been investigated. Materials considered include silicon dioxide, silicon nitride, aluminium nitride, alumina, and polycarbonate (Lexan). The passage of proton beams through ultrathin layers of these materials has been modeled by Monte Carlo simulations of particle transport. Parameters that have been varied in simulations include proton energy and insulating layer thickness. Materials are compared according to both ionizing and nonionizing effects produced by the passage of protons.

\section{Introduction}

Insulating layers are needed in various kinds of microelectronic components. Roles of these insulating layers vary from surface passivation of chips to more specific functions, such as lateral insulation of components in planar technology, capacitor dielectrics, or tunnel oxides in flash memory cells. Depending on their purposes within structures and devices, insulating layers are made out of different materials and with various thicknesses. Since many devices are expected to be operated in radiation environments, it is essential that radiation effects in insulators they comprise are investigated. Actual irradiation at accelerator facilities can be costly and time consuming, which is why radiation hardness of materials and components is often tested through simulations of radiation transport.

The present study investigates effects of proton beams on five insulating materials, commonly encountered in modern day electronic components: silicon dioxide, silicon nitride, aluminium nitride, alumina, and polycarbonate. Radiation effects are predicted and compared on the basis of Monte Carlo simulations of proton transport through ultrathin layers of these insulators.

\section{Radiation Effects in Insulators}

Insulators are a broad class of materials. They include crystals, amorphous materials, and organics (polymers). The response of insulators to irradiation is determined by their structural properties and electronic configuration $[1,2]$.

Interaction of incoming particles with matter results in two major effects: ionization energy loss and nonionizing energy loss (NIEL). Interactions of incoming particles which result in electronic excitation or ionization of atoms are referred to as ionization energy loss. In NIEL processes, the energy imparted by the incident particles results in atomic displacements or in collisions where the primary knock-on atom (PKA) remains in its lattice position, in which case the energy is converted to lattice vibrations (phonons). Displaced atoms can also undergo both electronic and displacement energy losses to dissipate their energy inside the medium.

Secondary electrons created by ionization energy losses affect electrical properties of crystalline insulators in a transient manner, except if these electrons get trapped at electrically active point defects in the crystal lattice. Point defects that serve as charge-carrier traps or donors, arise in irradiated insulators as a result of atomic displacements, that 
is, of NIEL. Polymer insulators exhibit radiation effects that are specific to that kind of materials. They include chain scission and cross-linking, both of which can significantly alter insulator's physical properties.

A heavy charged particle can transfer only a small fraction of its energy in a single electronic collision, and its deflection in the collision is negligible. It therefore has an almost straight trajectory in matter, losing energy continuously in small amounts through collisions with atomic electrons, leaving ionized and excited atoms along its path. Only occasionally does it undergo a substantial deflection, due to elastic scattering from an atomic nucleus [1-4].

For charged particles, such as protons, ionization, and electronic excitation, energy losses are represented by the electronic stopping power (also called collision stopping power, which is a misnomer, since all interactions can be considered collisions) of the material through which they propagate. Stopping power of a medium for a charged particle is the average linear rate of energy loss of the particle in the medium. It is, therefore, equal to the unrestricted linear energy transfer (LET). Several semiempirical stopping power formulas have been devised. The SRIM code, used for simulating proton transport in the present paper, implements the so-called ZBL stopping, which is based on the model given by Ziegler, Biersack, and Littmark.

Displacement damage can occur in crystal insulators when the energy transferred to lattice atoms exceeds the threshold displacement energy $\left(E_{d}\right)$. Irradiation of materials with electrons and light ions introduces predominantly isolated interstitial atoms and vacancies (Frenkel pairs) and small clusters of these point defects, because of the low average recoil atom energies $(0.1-1 \mathrm{keV})$. Energetic heavy ion irradiations, on the other hand, produce energetic displacement cascades that can lead to direct formation of defect clusters within isolated displacement cascades, due to more energetic average recoil atom energies $(>10 \mathrm{keV})[5,6]$.

The effects of irradiation on the electrical parameters of many materials have been found to display a simple, often linear, relationship with NIEL. NIEL is the rate at which energy is lost to nonionizing events. It is a direct analog of stopping power for ionization events. The units of NIEL are typically $\mathrm{MeV} / \mathrm{cm}$, or $\mathrm{MeV} \mathrm{cm} / \mathrm{g}$ if mass NIEL is considered. The calculation of NIEL requires information regarding the differential cross section for atomic displacements $(d \sigma / d \Omega)$, the average recoil energy of the target atoms $\left(E_{r}\right)$, and a term which partitions the energy into ionizing and nonionizing events, called the Lindhard partition factor $(L)$. NIEL can be written as an integral over solid angle as follows:

$$
\operatorname{NIEL}(E)=\frac{N_{A}}{A} \int_{\theta_{\min }}^{\pi} \frac{d \sigma(\theta, E)}{d \Omega} T(\theta, E) L(T(\theta, E)) d \Omega,
$$

where the $N_{A}$ is Avogadro's number, $A$ is the atomic mass, and $\theta_{\min }$ is the scattering angle for which the recoil energy equals the threshold for atomic displacement. For protons having nonrelativistic energy, the Rutherford differential cross section can be used for elastic scattering at atomic nuclei [7-11].

\section{Results of Simulations}

Monte Carlo simulations of proton transport through thin layers of insulators were performed in the TRIM module of the SRIM software package [12]. Simulations used monoenergetic unidirectional beams, incident perpendicularly on the film's surface. Proton energies were varied from $10 \mathrm{keV}$ to $10 \mathrm{MeV}$. At each value of proton energy, the thickness of the insulating film was increased until the whole beam was stopped within it. Film thickness in that case exceeded the maximum range of both protons and any secondary charged particles, which corresponds to a maximum radiation effect in the insulator. Each simulation run followed $10^{4}$ proton histories.

Results in Figures 1-5 present the most illustrative examples of proton beam effects in the studied insulators for the investigated proton energy range. Vacancy depth distribution plots, LET versus depth plots, and NIEL versus depth plots were obtained from SRIM outputs, following the procedure outlined in [13].

The rate of vacancy formation can be converted into NIEL using the modified Kinchin-Pease relationship between the number of atomic displacements $N_{d}$ and the nonionizing energy $E_{n}$ as follows:

$$
N_{d}=0.8 \frac{E_{n}}{2 E_{d}},
$$

where $E_{d}$ is the threshold energy for atomic displacement. Equation (2) applies for $E_{n}>2.5 E_{d}[13-15]$.

Distributions of atomic vacancies, created within the insulating films by the NIEL of both protons and recoils, are shown in total (curves denoted by "Total vacancies" in the graphs), but also for each kind of displaced atom separately (e.g., curves denoted by "Silicon vacancies" or "Oxygen vacancies"). Curves of total vacancy concentration along the depth of the insulating film are, naturally, always the top ones in these graphs.

\section{Analysis of the Results}

Thin insulating films investigated herein are immune to the passage of protons with energies exceeding $10 \mathrm{MeV}$. Simulation results (omitted from this paper) have shown that proton beams with $10 \mathrm{MeV}$ energy experience inconsiderable broadening (fanning) for the considered range of insulating film thickness (from $0.4 \mu \mathrm{m}$ to $20 \mu \mathrm{m}$ ). For these high energy protons, NIEL is also negligible, while LET is at least one order of magnitude lower than for $1 \mathrm{MeV}$ protons.

Of the four investigated insulators, protons went to larger penetration depths in silicon dioxide and lexan than in the other two materials. In $\mathrm{SiO}_{2}$, there is a somewhat greater concentration of silicon vacancies than oxygen ones, despite the $2: 1$ stoichiometric ratio of oxygen to silicon atoms, which is attributed to the threshold displacement energy of Si being considerably lower $\left(E_{d \mathrm{Si}}=15 \mathrm{eV}, E_{d \mathrm{O}}=28 \mathrm{eV}\right)$. Ionizing energy losses (LET) generally dominate NIEL by 1 to 3 orders of magnitude. This difference becomes larger as the energy of the proton beam is increased, which means that it subsides 


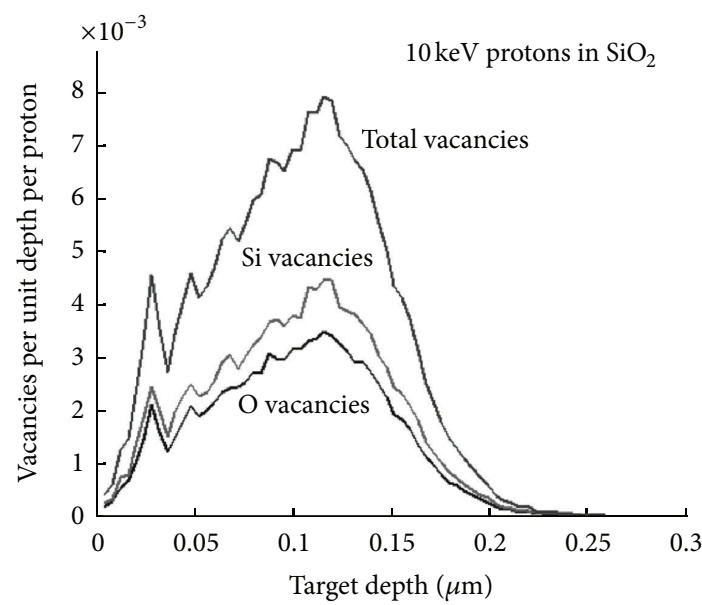

(a)

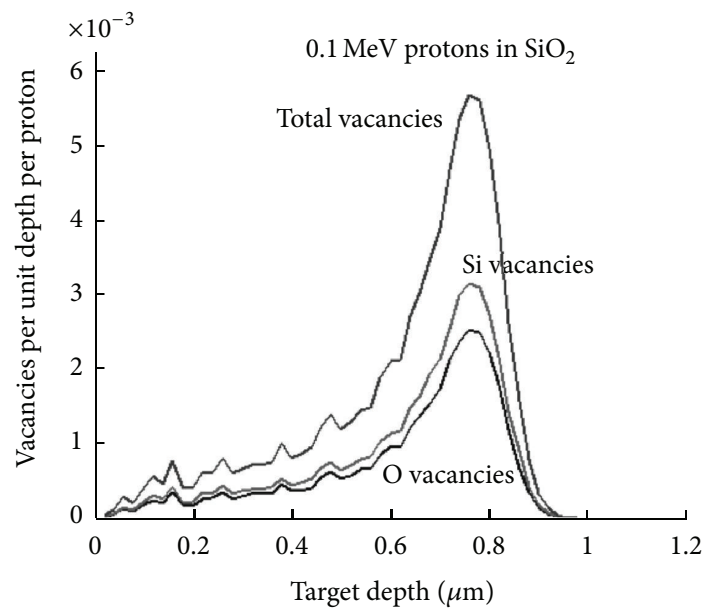

(c)

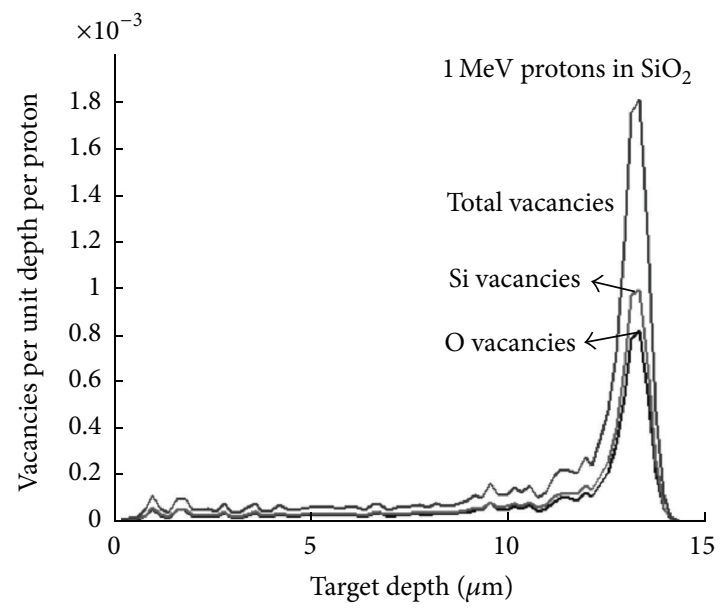

(e)

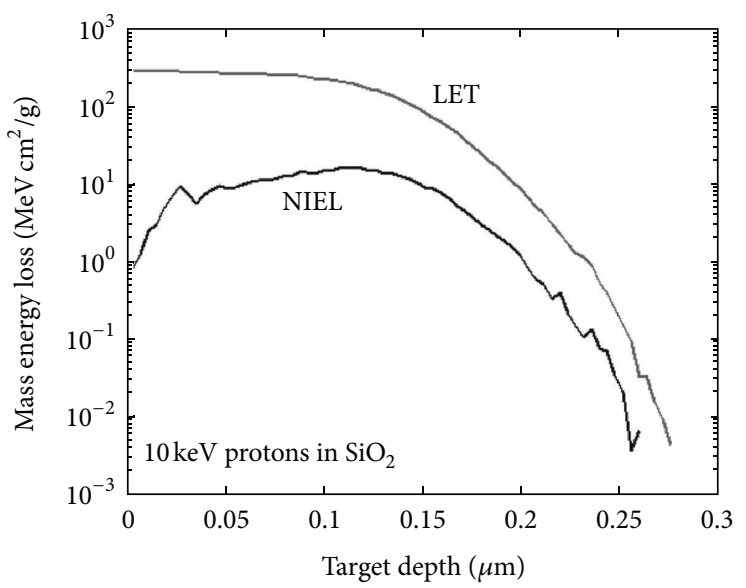

(b)

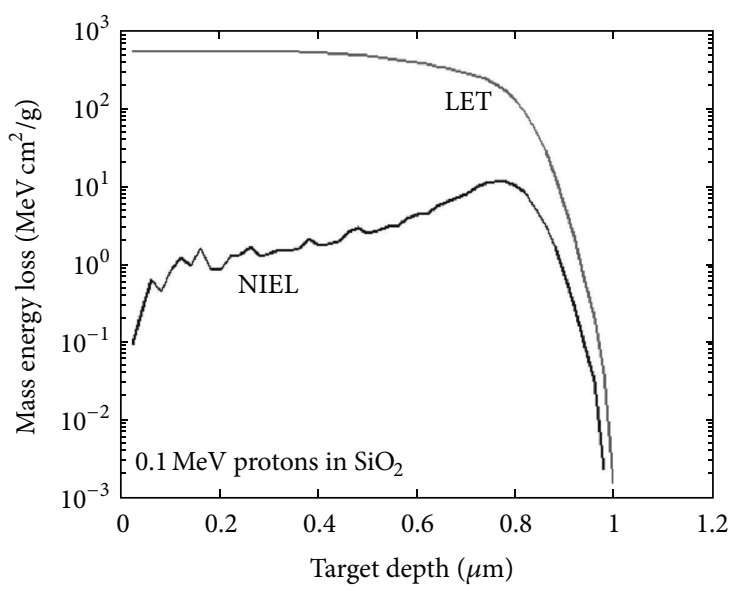

(d)

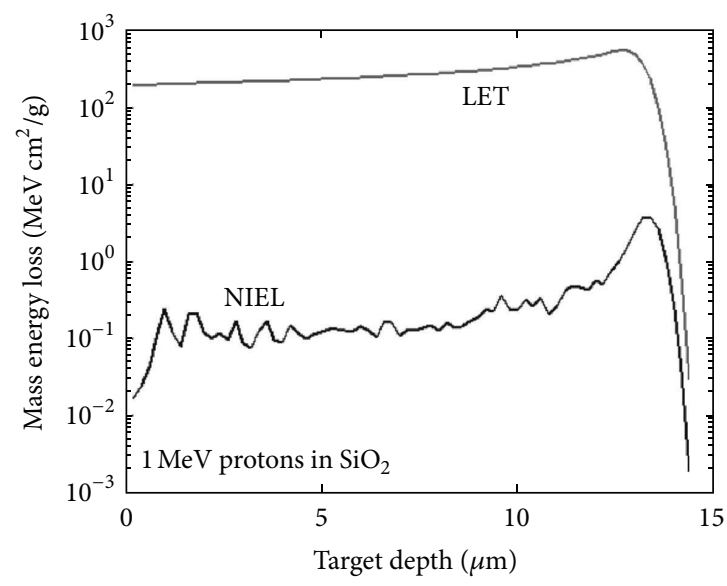

(f)

FIGURE 1: Plots of vacancy concentrations and mass energy losses (LET and NIEL divided by material density) along the depth of a silicon dioxide $\left(\mathrm{SiO}_{2}\right)$ insulating film. Results were obtained from simulations of $10^{4}$ proton histories, which included subcascades of recoiling atoms. 


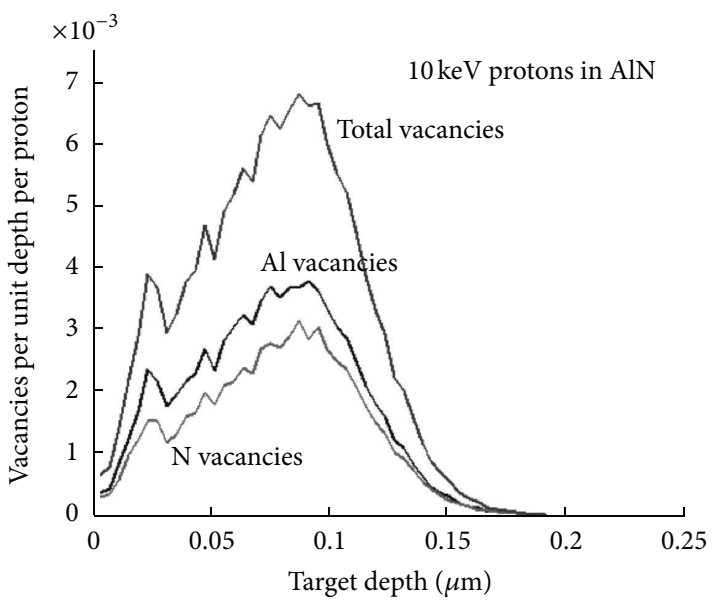

(a)

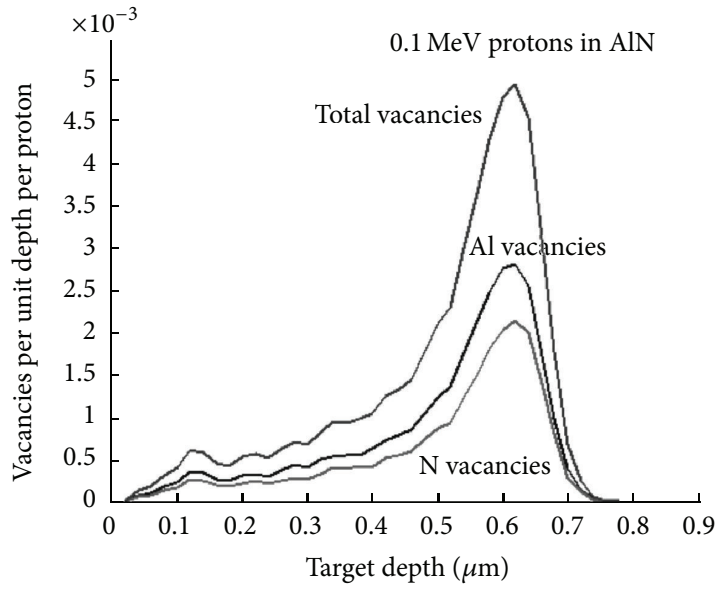

(c)

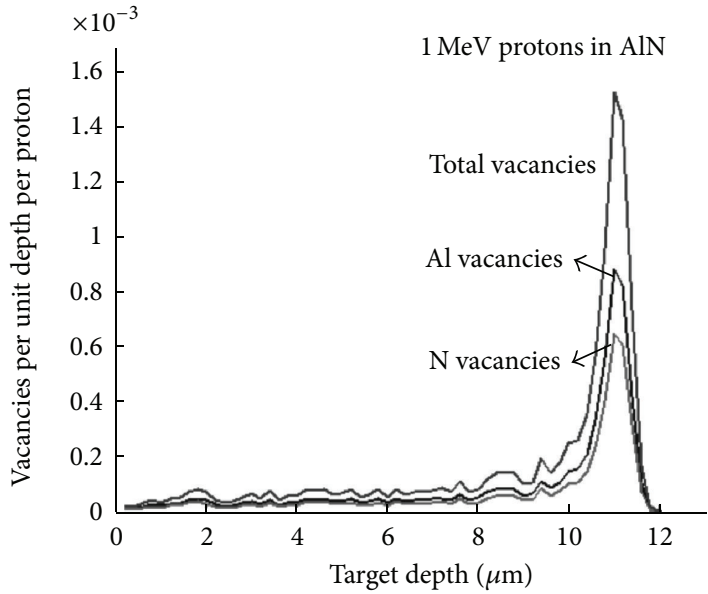

(e)

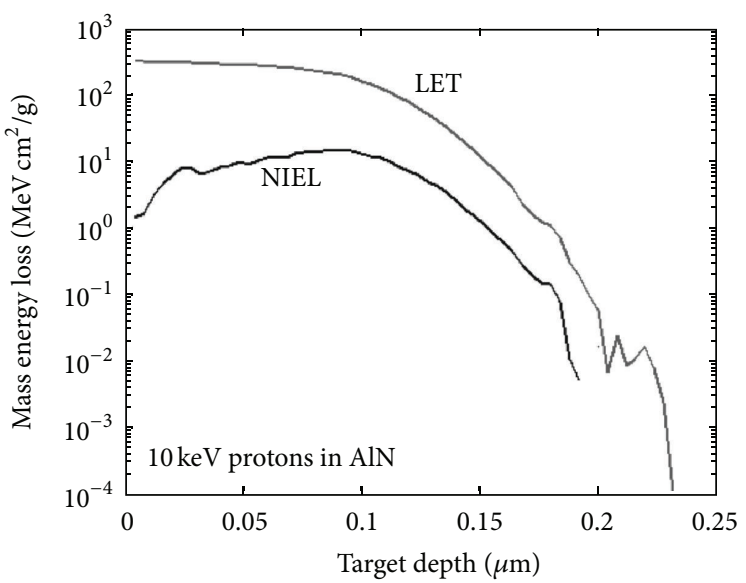

(b)

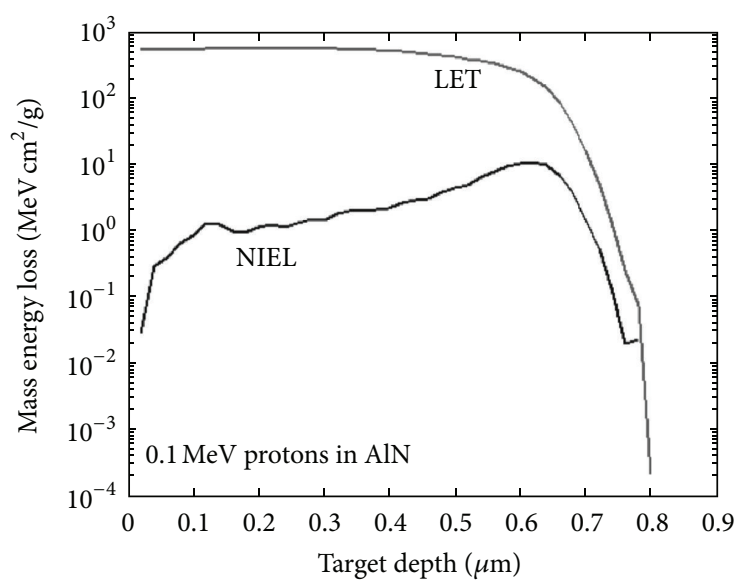

(d)

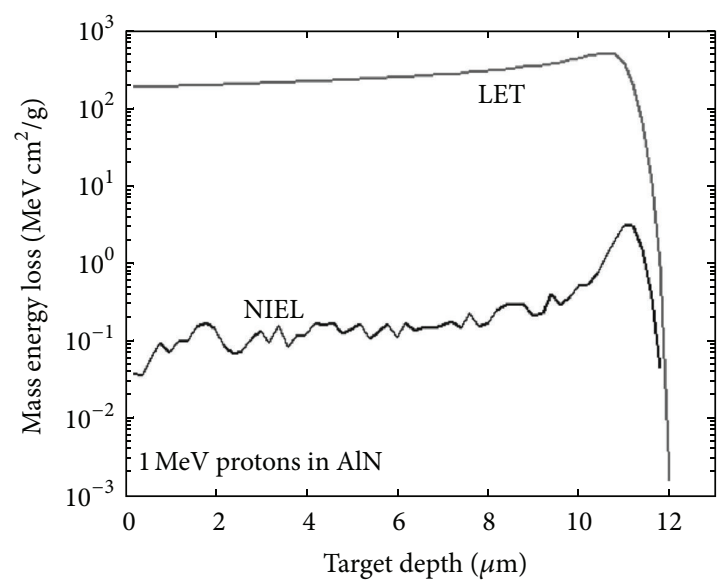

(f)

FIgURE 2: Plots of vacancy concentrations and mass energy losses (LET and NIEL divided by material density) along the depth of an aluminium nitride (AlN) insulating film. Results were obtained from simulations of $10^{4}$ proton histories, which included subcascades of recoiling atoms. 


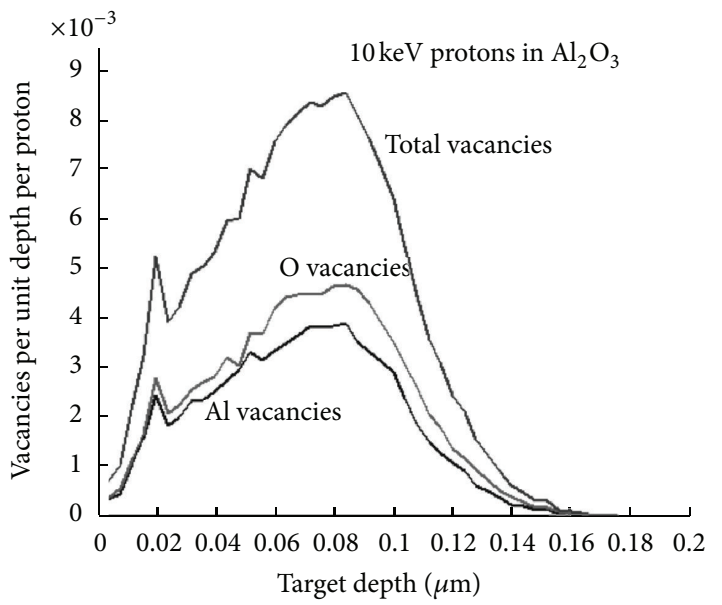

(a)

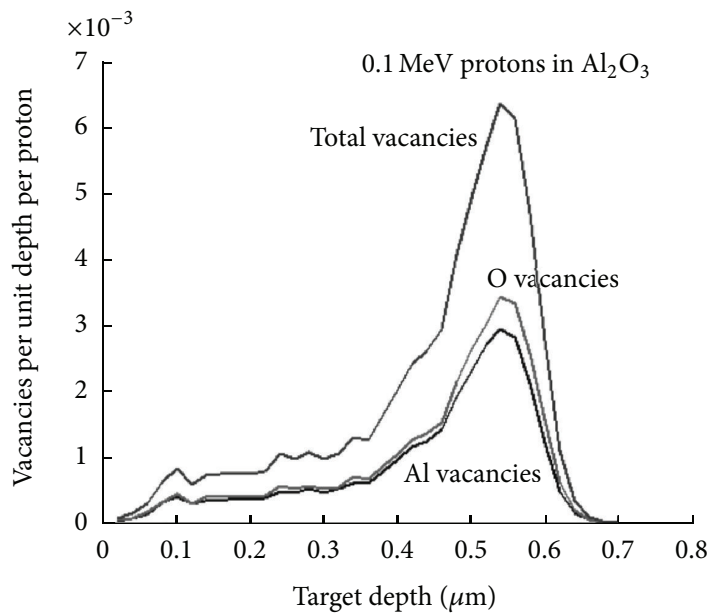

(c)

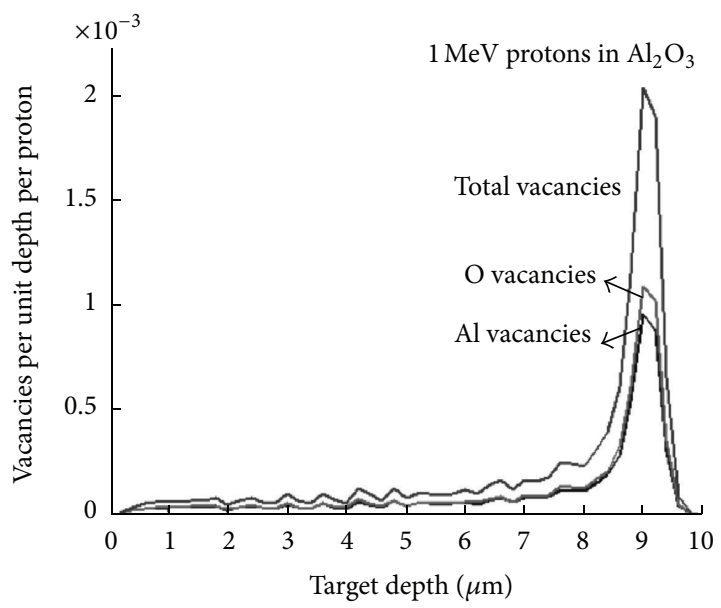

(e)

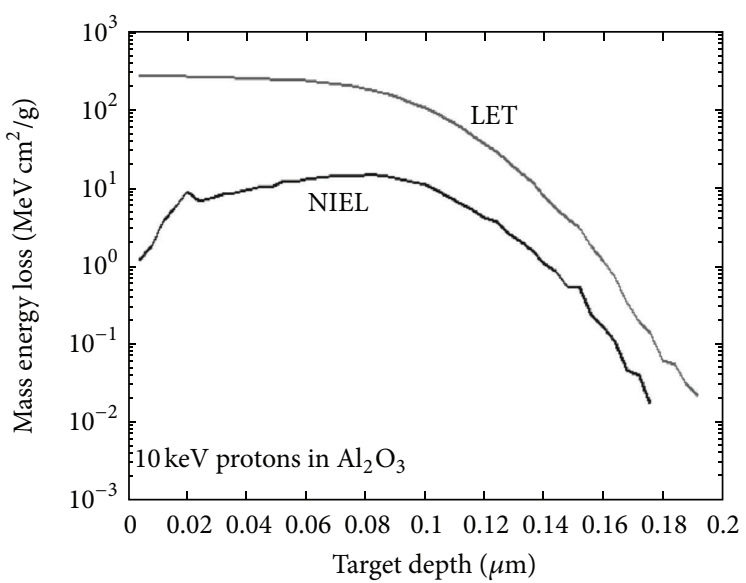

(b)

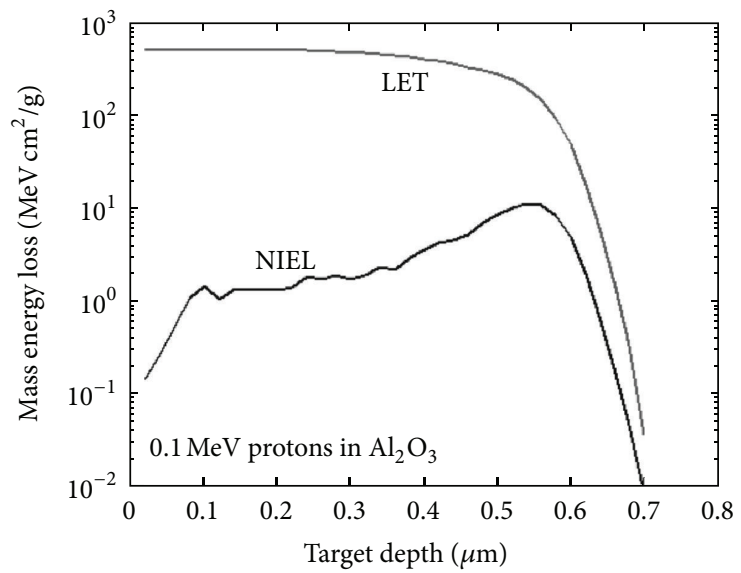

(d)

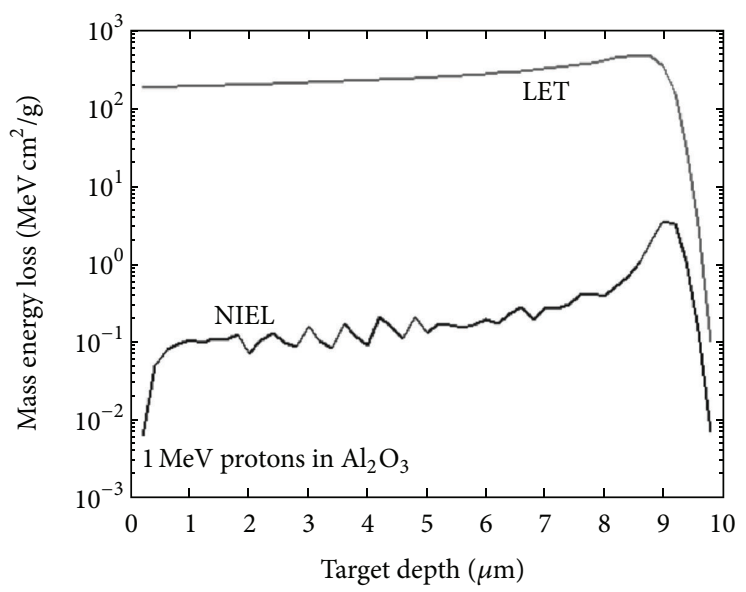

(f)

FIGURE 3: Plots of vacancy concentrations and mass energy losses (LET and NIEL divided by material density) along the depth of an alumina $\left(\mathrm{Al}_{2} \mathrm{O}_{3}\right)$ insulating film. Results were obtained from simulations of $10^{4}$ proton histories, which included subcascades of recoiling atoms. 


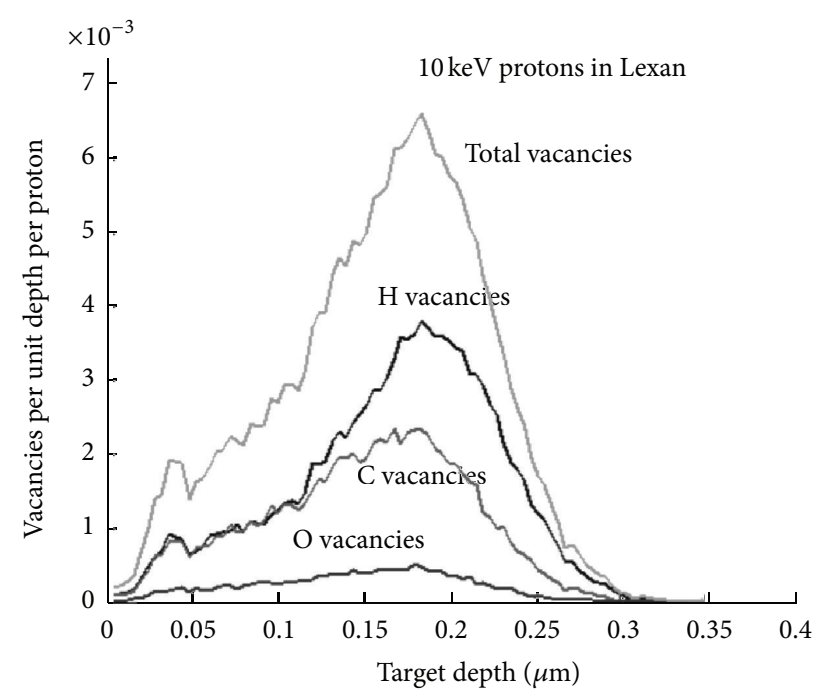

(a)

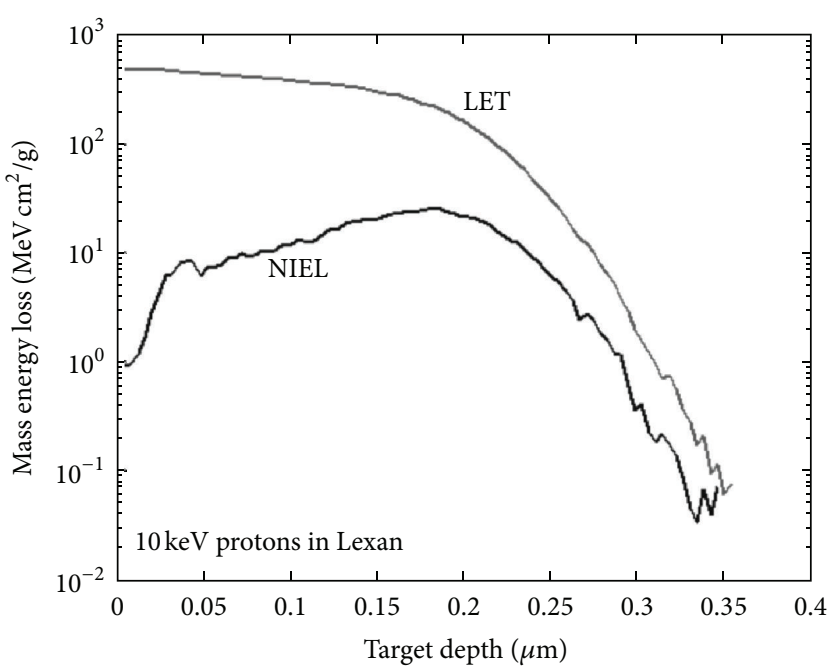

(b)

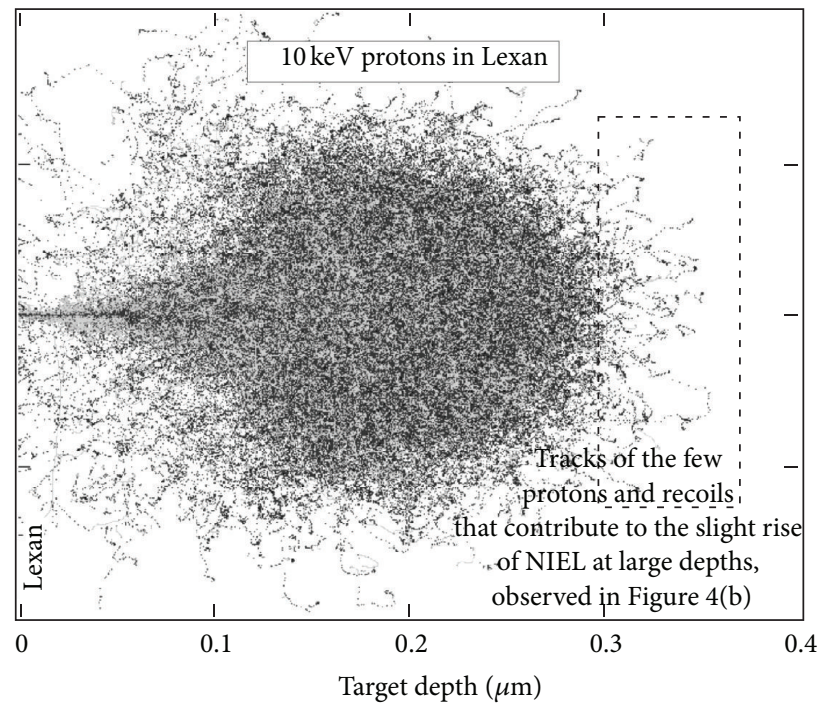

(c)

FIGURE 4: Plots of (a) vacancy concentrations and (b) mass energy losses (LET and NIEL divided by material density) along the depth of a $0.4 \mu \mathrm{m}$ thick lexan (polycarbonate) film. (c) Tracks of protons and recoiling atoms. Results were obtained from simulations of $10^{4}$ proton histories, for a $10 \mathrm{keV}$ incident proton beam.

towards the ends of proton tracks, where nuclear elastic scatterings that give rise to atomic displacements become more probable than electronic collisional events.

The slight increase of NIEL seen at the rightmost part of the lower curve in Figure 4(b) is due to the few protons that reach penetration depths close to $0.35 \mu \mathrm{m}$, larger than the mean beam range, and also due to the recoils (mainly hydrogen) that these protons displace. Although the number of such protons is small, they arrive at these large depths without losing much of the original energy in electronic collisions, as most other protons from the beam do. The resulting rise of the NIEL curve is small, but still noticeable in the logarithmic scale. Tracks of protons and recoils that contribute to this rise of NIEL are marked in Figure 4(c).
The energy loss versus depth plot begins to take the classic shape of a Bragg curve only at proton energies $>0.5 \mathrm{MeV}$. As proton energy is increased from $\sim 100 \mathrm{keV}$, it is first the NIEL curve that starts exhibiting a peak near the maximum penetration depth, as seen in Figures 1(d), 2(d), and 3(d). Around $1 \mathrm{MeV}$ proton energy, both LET and NIEL curves peak toward the end of the penetration depth, giving shape to the Bragg peak, observed much more clearly when the scale of the mass energy loss axis is linear, and not logarithmic. This is illustrated by the plot in Figure 6 for $1 \mathrm{MeV}$ protons in $\mathrm{SiO}_{2}$.

If one cascade creates more than 8000 recoil atoms, SRIM discards the atoms beyond 8000 , which causes certain inaccuracy in vacancy calculations. During our simulations, this happened for protons with energies higher than $\sim 1 \mathrm{MeV}$ 


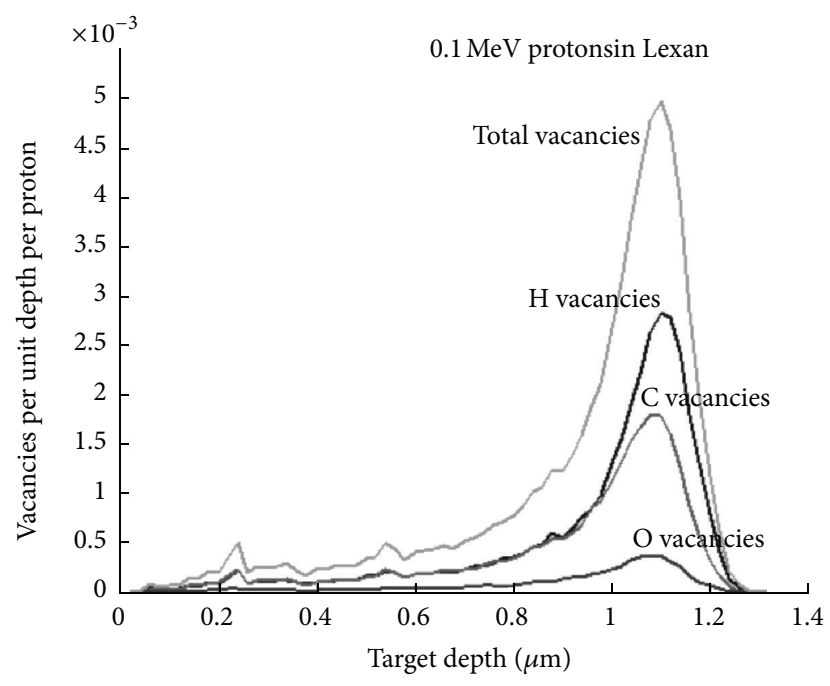

(a)

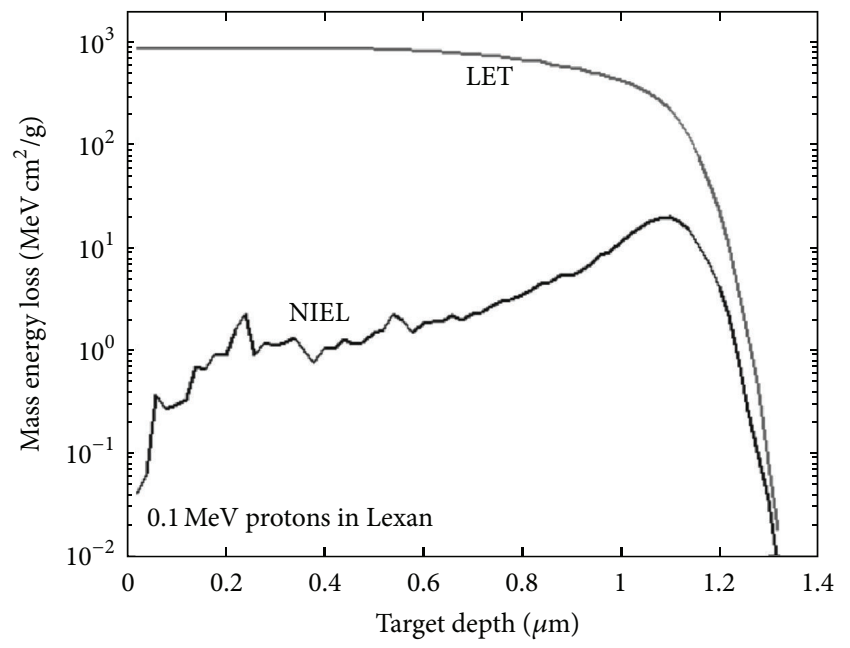

(b)

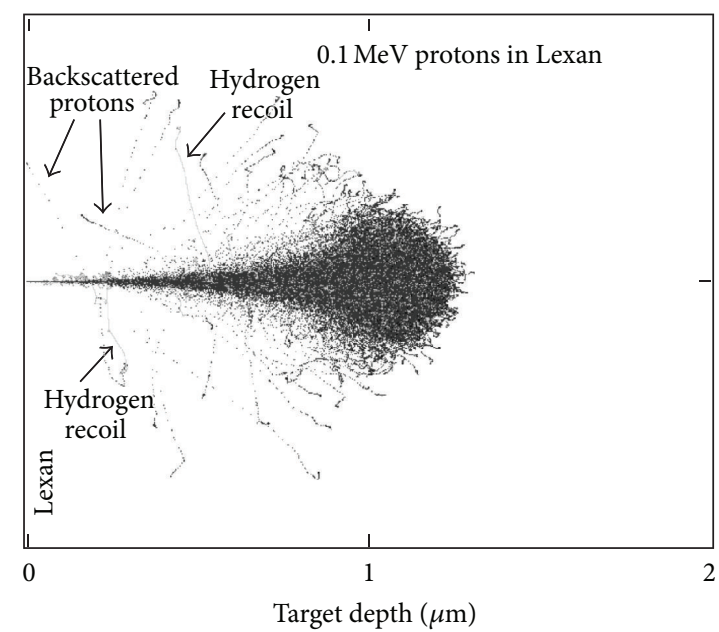

(c)

FIGURE 5: Plots of (a) vacancy concentrations and (b) mass energy losses (LET and NIEL divided by material density) along the depth of a $0.2 \mu \mathrm{m}$ thick lexan (polycarbonate) film. (c) Tracks of protons and recoiling atoms. Results were obtained from simulations of $10^{4}$ proton histories, for a $0.1 \mathrm{MeV}$ incident proton beam.

in lexan films. In a hydrogen-rich medium such as lexan, high energy incident protons give rise to a large number of energetic hydrogen knockons, which results in a multitude of highly branched cascades.

Figure 5(c) clearly demonstrates large-angle backscattering of protons and long tracks of energetic hydrogen recoils in lexan.

Chemical structure unit of polycarbonate is presented in Figure 7. It contains 14 atoms of hydrogen, 16 of carbon, and 3 of oxygen. The stoichiometric ratio of the three elements, along with their threshold displacement energies $\left(E_{d \mathrm{H}}=\right.$ $10 \mathrm{eV}, E_{d \mathrm{C}}=E_{d \mathrm{O}}=28 \mathrm{eV}$ ), accounts for the observed ratios of vacancy concentration curves seen in Figures 4(a) and 5(a).

Secondary electrons, produced by protons through ionization energy losses, further interact with polymer macromolecules, causing their ionization and excitation. Relaxation of excited molecules and locally formed ionization clusters results in a formation of large amounts of free radicals. Highly reactive free radicals cause destruction of polymer chains, by either chain scission (random rupturing of bonds) or crosslinking (formation of large three-dimensional molecular networks). As a result of chain scission, low-molecularweight fragments, gas evolution, and unsaturated bonds may appear [16].

Many important physical and chemical properties of polymers can be modified by irradiation. Among these are molecular weight, chain length, entanglement, polydispersity, branching, and chain termination. These structural changes also affect the electrical insulating properties of polycarbonate films $[17,18]$.

Radiation effects in thin insulating layers are relevant due to the increasing miniaturization of electronic components. Miniaturization makes these components sensitive to primary and secondary cosmic rays, which also present one of 


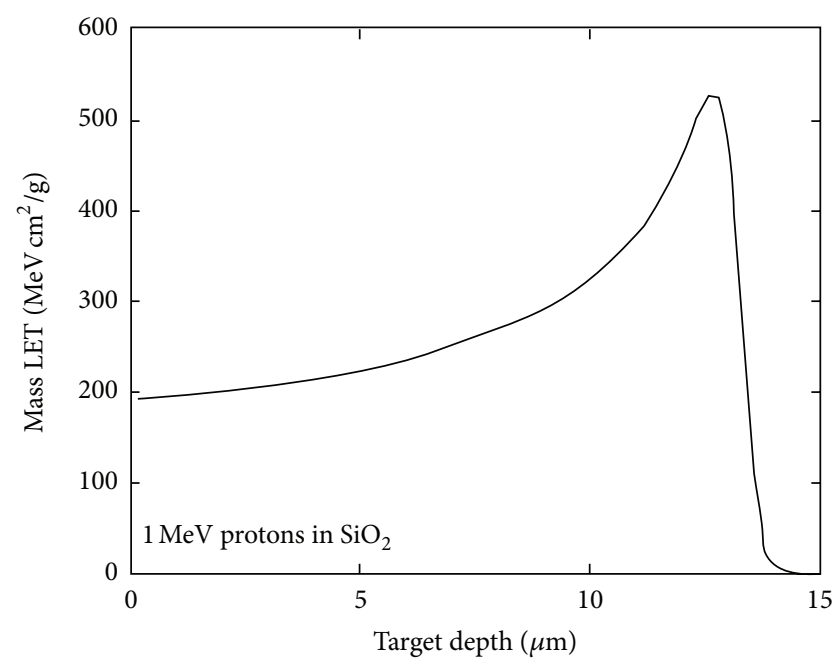

FIGURE 6: Plot of the mass LET for $1 \mathrm{MeV}$ protons along the depth of a $15 \mu \mathrm{m}$ thick silicon dioxide film. The scale of the energy loss axis is linear, in contrast to Figure 1(f) where it is logarithmic.

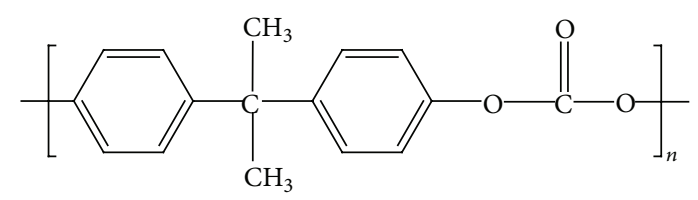

Figure 7: Chemical structure unit of polycarbonate, containing 14 atoms of hydrogen, 16 of carbon, and 3 of oxygen.

the most severe obstacles for a widespread use of photovoltaic cells [19-22].

\section{Conclusion}

Monte Carlo simulations of proton transport through thin films of $\mathrm{SiO}_{2}, \mathrm{AlN}, \mathrm{Al}_{2} \mathrm{O}_{3}$ and polycarbonate have shown that the investigated films are immune to the passage of protons with energies higher than $\sim 10 \mathrm{MeV}$. Nonionizing energy loss of these high energy protons is low, and they traverse the films without much atomic displacement. In the lower part of the investigated proton energy range (from $10 \mathrm{keV}$ to $1 \mathrm{MeV}$ ), however, substantial ionization losses and NIEL are to be expected. Ionization and displacement damage produced by protons could influence the properties of these insulators and compromise their reliability within complex structures and devices. Point defects, some of which are charge-carrier donors, arise in irradiated insulators as a result of atomic displacements. Highly reactive free radicals that can appear in irradiated lexan cause chain scission and/or cross-linking, which then affects the insulating properties of polycarbonate films.

\section{Acknowledgment}

The Ministry of Education and Science of the Republic of Serbia supported this work under Contract 171007.

\section{References}

[1] A. G. Holmes-Siedle and L. Adams, Handbook of Radiation Effects, Oxford University Press, 2nd edition, 2002.

[2] J. R. Schwank, M. R. Shaneyfelt, D. M. Fleetwood et al., "Radiation effects in MOS oxides," IEEE Transactions on Nuclear Science, vol. 55, no. 4, pp. 1833-1853, 2008.

[3] S. Stanković, B. Iričanin, D. Nikolić et al., "MSV signal processing system for neutron-gamma discrimination in a mixed field," Nuclear Technology \& Radiation Protection, vol. 27, no. 2, pp. 165-170, 2012.

[4] I. Knezevic, N. Zdjelarevic, M. Obrenovic, and M. Vujisic, "Absorbed dose assessment in particle-beam irradiated metaloxide and metal-nonmetal memristors," Nuclear Technology \& Radiation Protection, vol. 27, no. 3, pp. 290-296, 2012.

[5] P. Sigmund, Stopping of Heavy Ions, vol. 4 of Springer Tracts of Modern Physics, Springer, Berlin, Germany, 2004.

[6] J. H. Warner, S. R. Messenger, R. J. Walters, and G. P. Summers, "Displacement damage correlation of proton and silicon ion radiation in GaAs," IEEE Transactions on Nuclear Science, vol. 52, no. 6, pp. 2678-2682, 2005.

[7] H. García, S. Dueñas, H. Castán et al., "Irradiation effect on dielectric properties of hafnium and gadolinium oxide gate dielectrics," Journal of Vacuum Science and Technology B, vol. 27, no. 1, pp. 416-420, 2009.

[8] C. Leroy and P.-G. Rancoita, "Particle interaction and displacement damage in silicon devices operated in radiation environments," Reports on Progress in Physics, vol. 70, no. 4, pp. 493-625, 2007.

[9] R. Radosavljević and A. Vasić, "Effects of radiation on solar cells as photovoltaic generators," Nuclear Technology \& Radiation Protection, vol. 27, no. 1, pp. 28-32, 2012.

[10] Đ. Lazarević, M. Vujisić, K. Stanković, E. Dolićanin, and P. Osmokrović, "Radiation hardness of indium oxide films in the cooper-pair insulator state," Nuclear Technology \& Radiation Protection, vol. 27, no. 1, pp. 40-43, 2012.

[11] E. Dolićanin, "Gamma ray effects on flash memory cell arrays," Nuclear Technology \& Radiation Protection, vol. 27, no. 3, pp. 284-289, 2012.

[12] J. F. Ziegler, J. P. Biersack, and M. D. Ziegler, "SRIM-The Stopping and Range of Ions in Matter," http://www.srim.org.

[13] S. R. Messenger, E. A. Burke, G. P. Summers et al., "Nonionizing Energy Loss (NIEL) for heavy ions," IEEE Transactions on Nuclear Science, vol. 46, pp. 1595-1602, 1999.

[14] D. Nikolić, A. Vasić, I. Fetahović, K. Stanković, and P. Osmokrović, "Photodiode behavior in radiation environment," Applied Mathematics, Informatics \& Mechanics, vol. 3, no. 1, pp. 27-34, 2011.

[15] M. Vujisić, N. Marjanović, I. Fetahović, K. Stanković, and P. Osmokrović, "Influence of radiation on titanium dioxide mermistors," Applied Mathematics, Informatics \& Mechanics, vol. 4, no. 1, pp. 75-82, 2012.

[16] "Polymer durability and radiation effects," in Proceedings of the Acs Symposium, M. C. Celina and R. A. Assink, Eds., series 978, 2007.

[17] M. P. Petkov, K. G. Lynn, K. P. Rodbell, W. Volksen, and R. D. Miller, "Radiation effects in low dielectric constant methylsilsesquioxane films," IEEE Transactions on Nuclear Science, vol. 49 , no. 6 , pp. 2724-2728, 2002.

[18] T. Iwahashi, T. Tamagawa, A. Hayato et al., "Heavy ion and proton irradiation of gas electron multipliers with liquid crystal 
polymer insulator: evaluation tests for use in space," IEEE Transactions on Nuclear Science, vol. 58, no. 5, pp. 2401-2406, 2011.

[19] X. L. Han, H. Ni, K. Zhao, Y.-C. Kong, H. K. Wong, and S. Q. Zhao, "Load-resistance- and voltage-tunable photovoltaic effect in tilting manganite films," International Journal of Photoenergy, vol. 2011, Article ID 314085, 2011.

[20] Z. Hu, B. Jiao, J. Zhang, X. Zhang, and Y. Zhao, "Indium-doped zinc oxide thin films as effective anodes of organic photovoltaic devices," International Journal of Photoenergy, vol. 2011, Article ID 158065, 5 pages, 2011.

[21] T. T. Chow, G. N. Tiwari, and C. Menezo, "Hybrid Solar: a review on photovoltaic and thermal power integration," International Journal of Photoenergy, vol. 2012, Article ID 307287, 17 pages, 2012.

[22] P. Ooshaksaraei, K. Sopian, R. Zulkifli, M. A. Alghoul, and S. H. Zaidi, "Characterization of a bifacial photovoltaic panel integrated with external diffuse and semimirror type reflectors," International Journal of Photoenergy, vol. 2013, Article ID 465837, 7 pages, 2013. 

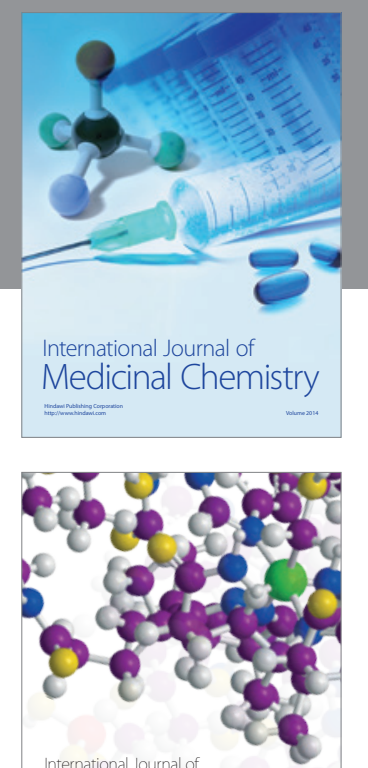

\section{Carbohydrate} Chemistry

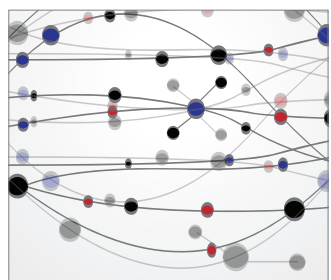

The Scientific World Journal
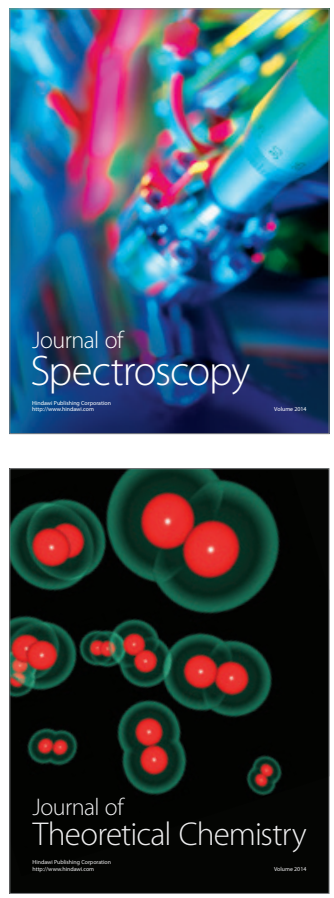
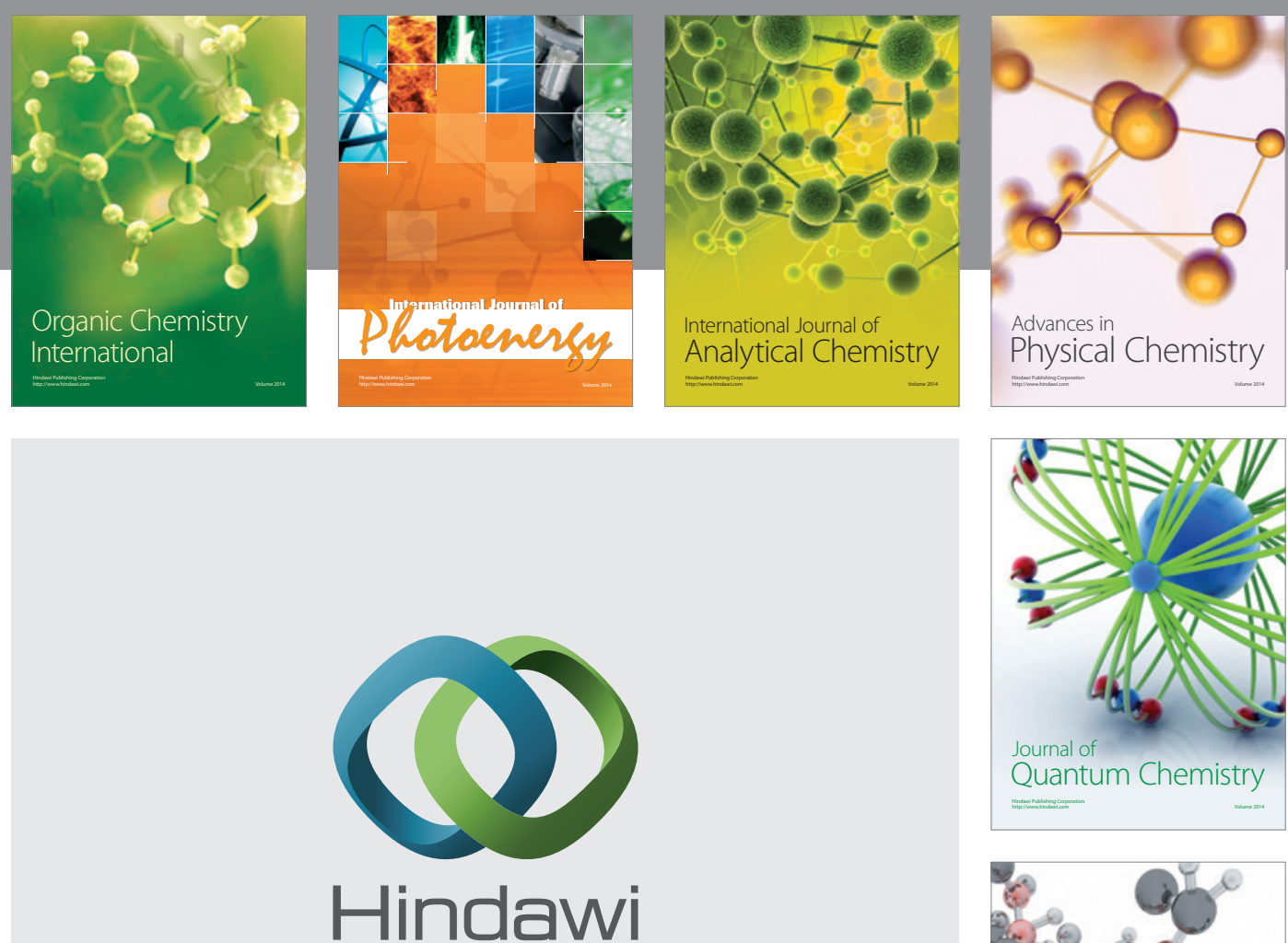

Submit your manuscripts at

http://www.hindawi.com

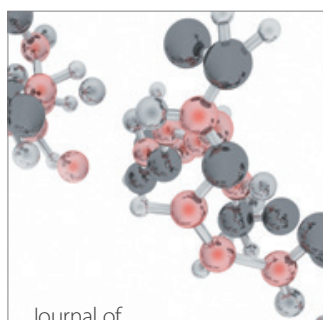

Analytical Methods

in Chemistry

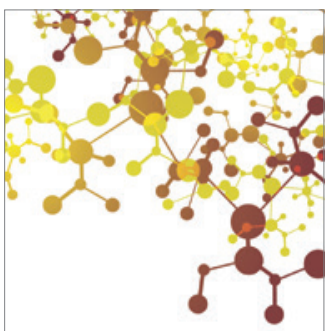

Journal of

Applied Chemistry

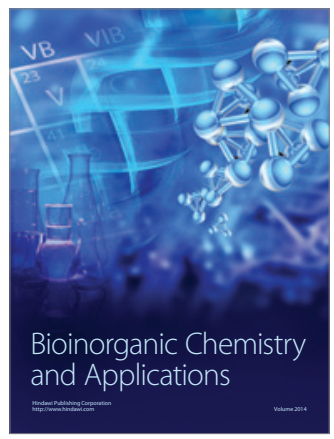

Inorganic Chemistry
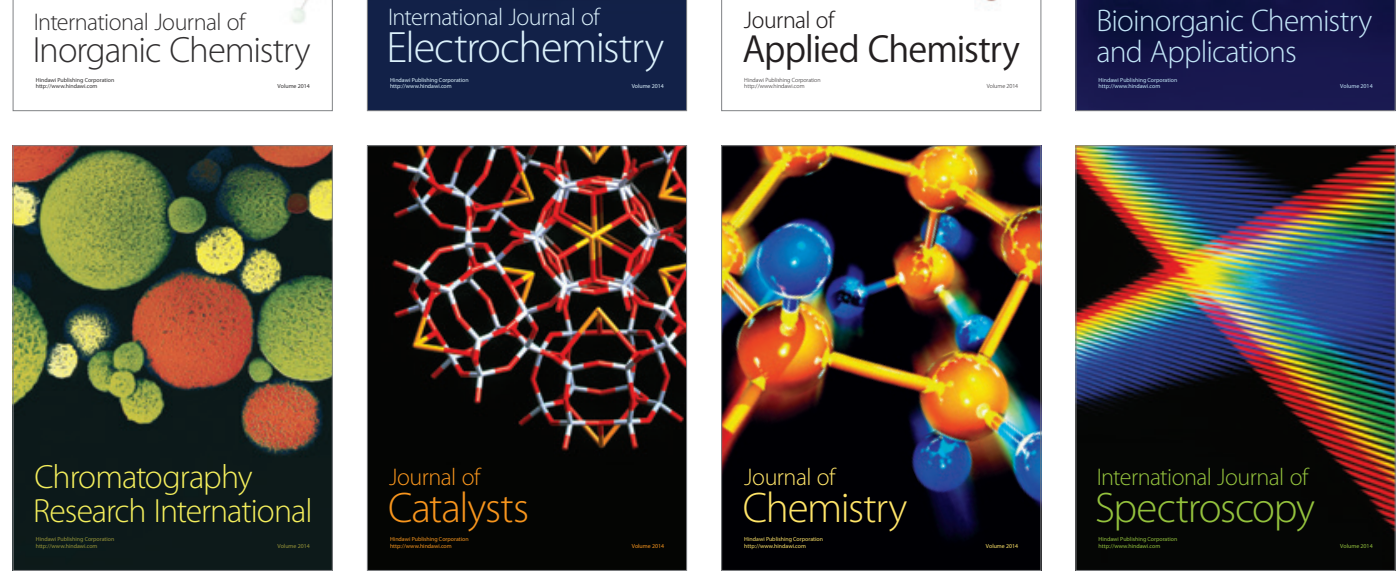